\title{
TGF- $\alpha$ Overexpression in Breast Cancer Bone Metastasis and Primary Lesions and TGF- $\alpha$ Enhancement of Expression of Procancer Metastasis Cytokines in Bone Marrow Mesenchymal Stem Cells
}

\author{
Jingbo Sun, ${ }^{1}$ Haiyan Cui, ${ }^{2}$ Yanxin Gao, ${ }^{1}$ Yangjian Pan, ${ }^{1}$ Kun Zhou, ${ }^{1}$ Jingzhan Huang, \\ Jin Lan, ${ }^{1}$ Qingzhu Wei, ${ }^{3}$ Xiaolong Liu ${ }^{\mathbb{D}},{ }^{1}$ Lixin Liu $\left(\mathbb{D},{ }^{1}\right.$ and Cory J. Xian $\mathbb{D}^{4}$ \\ ${ }^{1}$ Department of General Surgery, The Third Affiliated Hospital of Southern Medical University, Guangzhou, Guangdong 510630, China \\ ${ }^{2}$ Department of Internal Medicine, The Third Affiliated Hospital of Southern Medical University, Guangzhou, \\ Guangdong 510630, China \\ ${ }^{3}$ Department of Pathology, The Third Affiliated Hospital of Southern Medical University, Guangzhou, Guangdong 510630, China \\ ${ }^{4}$ Sansom Institute for Health Research, School of Pharmacy and Medical Sciences, University of South Australia, Adelaide, \\ SA 5001, Australia \\ Correspondence should be addressed to Xiaolong Liu; lxl1979@i.smu.edu.cn and Lixin Liu; dr.lixin.liu@hotmail.com
}

Received 24 October 2017; Revised 29 November 2017; Accepted 27 December 2017; Published 8 February 2018

Academic Editor: Cho-Pei Jiang

Copyright (c) 2018 Jingbo Sun et al. This is an open access article distributed under the Creative Commons Attribution License, which permits unrestricted use, distribution, and reproduction in any medium, provided the original work is properly cited.

\begin{abstract}
Bone metastasis (BM) is the advanced complication of breast cancer, while bone marrow-derived mesenchymal stem cells (BMSCs) in the microenvironment unclearly contribute to cancer metastasis. This study investigated potential roles of transforming growth factor- (TGF-) $\alpha$ in the interaction between breast cancer and BMSCs in BM. Clinical cases of breast cancer with bone metastasis $(\mathrm{BMBC})$, breast cancer without bone metastasis (Non-BM-BC), and benign fibroadenoma (Benign) were enlisted in a retrospective study. TGF- $\alpha$ was found obviously overexpressed in BM lesion of BMBC compared to primary lesion of both BMBC and NonBM-BC $(P<0.01)$, and TGF- $\alpha$ was higher in primary lesion of both BMBC and Non-BM-BC $(P<0.01)$ than Benign group. Interestingly, TGF- $\alpha$ in nontumor tissues of both BMBC and Non-BM-BC was at a higher level than Benign group $(P<0.01)$, and numbers of macrophages in nontumor tissues of both BMBC and Non-BM-BC $(P<0.01)$ were higher than Benign group. Furthermore, in cultured human BMSCs, TGF- $\alpha$ stimulated production of procancer cytokines including IL-6, VEGF, FGF10, FGF17, and TGF- $\beta 1$ in a dose-dependent manner. Thus, TGF- $\alpha$ in BC could potentially be an important signal of carcinogenesis and metastasis. Macrophages in the nontumor tissue of $\mathrm{BC}$ may not be protective but could promote cancer metastasis.
\end{abstract}

\section{Introduction}

Breast cancer $(\mathrm{BC})$ is the number 1 malignant tumor among females worldwide, and malignant metastasis is the major cause of breast cancer related death. About 70\% BC cases are known to develop bone metastasis, and even $20 \%$ BC cases have bone metastasis in the early stage of primary lesion $[1,2]$. Bone metastasis breast cancer (BMBC) is a complicated and advanced stage that starts in the primary tumor microenvironment. Tumor microenvironment is composed of tumor cells, mesenchymal stem cells (MSCs), fibroblasts, immune cells, vascular endothelial cells, other stromal cells, and extracellular matrix [3]. It has been accepted that the interaction between $\mathrm{BC}$ cells and components in the microenvironment could influence the progression of BC [4].

MSCs are multipotent stem cells that are mainly derived from bone marrow, fat tissue, umbilical cord blood, and dental pulp [5-7]. Bone marrow-derived MSCs (BMSCs) are progenitors of fibroblasts, adipocytes, osteoblasts, and cartilage cells in normal human microenvironment and play important roles in tissue regeneration $[8,9]$. In the tumor microenvironment, BMSCs produce cytokines and exosomes 
TABLE 1: Clinical data of the four bone metastasis breast cancer cases.

\begin{tabular}{|c|c|c|c|c|}
\hline & Case 1 & Case 2 & Case 3 & Case 4 \\
\hline Gender & Female & Female & Female & Female \\
\hline Age & 46 & 66 & 71 & 46 \\
\hline Pathology subtypes & $\begin{array}{l}\text { Invasive ductal } \\
\text { carcinoma }\end{array}$ & Invasive ductal carcinoma & $\begin{array}{l}\text { Invasive lobular } \\
\text { carcinoma }\end{array}$ & $\begin{array}{l}\text { Invasive ductal } \\
\text { carcinoma }\end{array}$ \\
\hline Pathological grade & III & III & III & III \\
\hline Molecular subtypes & Luminal B & Luminal B & Luminal B & Luminal B \\
\hline $\begin{array}{l}\text { TNM stage, tumor } \\
\text { max diameter }(\mathrm{cm})\end{array}$ & IV, T: 2.2 & IV, T: 2.5 & IV, T: 3.3 & IV, T: 3.7 \\
\hline $\begin{array}{l}\text { Location of } \\
\text { metastasis }\end{array}$ & $\begin{array}{l}\text { Acetabular bone; } \\
\text { axillary lymph node }\end{array}$ & Thighbone & $\begin{array}{l}\text { Humerus; femur; liver; } \\
\text { axillary lymph node }\end{array}$ & $\begin{array}{l}\text { Blade bone; thighbone; } \\
\text { axillary lymph node }\end{array}$ \\
\hline $\begin{array}{l}\text { Remote metastasis } \\
\text { organ number }\end{array}$ & One & One & Three & Two \\
\hline
\end{tabular}

to promote tumor invasion and metastasis. It has been shown that plasminogen activator inhibitor- (PAI-) 1, interleukin(IL-) 6, Notch 1, and CD44 produced from BMSCs improve colorectal cancer cell survival and promote its development [10]. IL-6 and vascular endothelial growth factor (VEGF) from BMSCs improve BC metastasis, and the phenomenon was enhanced when BC cells are exposed to IL-6 and VEGF together [11]. In three-dimensional culture, BC cells developed invasion capacity when treated with transforming growth factor- (TGF-) $\beta 1$ from BMSCs [12]. BC cells have been reported to be able to capture and cannibalize BMSCs in the microenvironment which can enable them to change into a dormant state that can increase drug resistance and immunosuppression and finally increase cancer recurrence [13].

Transforming growth factor- (TGF-) $\alpha$ was reported by bioinformatic analyses to potentially stimulate BMSCs to promote breast cancer [14]. TGF- $\alpha$ is a transforming growth factor as part of a human 160-amino-acid transmembrane precursor, and it is a ligand for the epidermal growth factor receptor which plays roles in tissue regeneration and bone homeostasis and may promote tumorigenesis [15-17]. Elevated TGF- $\alpha$ is associated with the tumorigenesis of the breast and stomach $[18,19]$. However, whether TGF- $\alpha$ has a role in stimulating BMSCs during breast cancer bone metastasis still remains unclear.

In this study, as steps for identifying the role of TGF$\alpha$ in breast cancer bone metastasis, expression of TGF$\alpha$ in primary lesion and bone metastasis of breast cancer was analyzed and the influence of TGF- $\alpha$ on BMSCs was evaluated.

\section{Method and Materials}

2.1. Human Cases. Informed consent from all the patients was obtained and the study was approved by Ethics Board of the Third Affiliated Hospital of Southern Medical University.

2.1.1. Breast Cancer with Bone Metastasis (BMBC) Group. Four female breast cancer cases with bone metastasis were enrolled in the retrospective study as the observation group.
The 4 cases were diagnosed with breast cancer bone metastasis and received both mastectomy and excision of bone metastasis. The rule-in criteria also included no involvements of central nervous system diseases, gynecological diseases, or autoimmune diseases that might influence breast cancer progression and TGF- $\alpha$ expression. Their primary lesions were in the early stage of the TNM staging system [20]. The clinical data of these 4 BMBC cases were listed in Table 1 and shown in Figure 1.

2.1.2. Breast Cancer without Bone Metastasis (Non-BM-BC) Control Group. Four female breast cancer cases without bone metastasis were enrolled in the study as Non-BM-BC control group. The 4 cases were diagnosed with resectable breast cancer without bone metastasis (Non-BM-BC) and received mastectomy. The rule-in criteria included age being above 40 years to balance the influence of age, specific pathological diagnosis results (nonspecific invasive ductal carcinoma, WHO grade III; primary lesion, T2; and molecular group: luminal B), and no involvements of central nervous system diseases, gynecological diseases, or autoimmune diseases that might influence breast cancer progression and TGF- $\alpha$ expression. Their primary lesions were in the early stage of TNM staging.

2.1.3. Benign Control Group. Four female breast fibroadenoma cases were enlisted in the study as the negative control group. The 4 cases were diagnosed with potentially malignant breast lump before operation and received lumpectomy. The postoperative pathological results showed breast fibroadenoma. The rule-in criteria included age above 40 years and no involvements of central nervous system diseases, gynecological diseases, or autoimmune diseases. Their primary lesions were in the early stage of TNM staging.

2.2. Immunohistochemical Analyses (IHC). Samples of primary lesions and bone metastasis were fixed in formalin and embedded in paraffin and analyzed by immunohistochemical analysis. The $4 \mu \mathrm{m}$ sections were deparaffinized and rehydrated, and their endogenous peroxidase activity was inhibited with $0.3 \% \mathrm{H}_{2} \mathrm{O}_{2}$ methanol. After being 


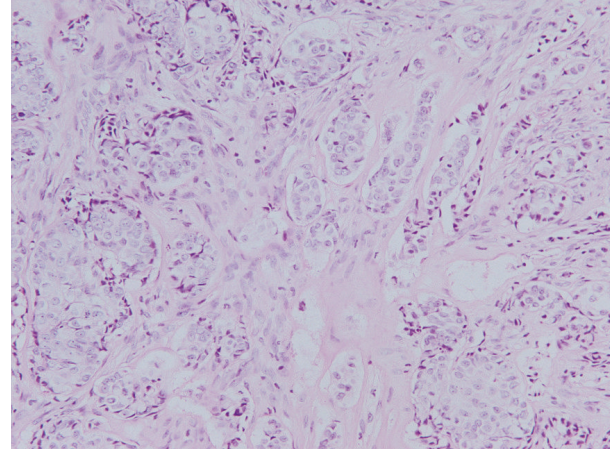

(a)

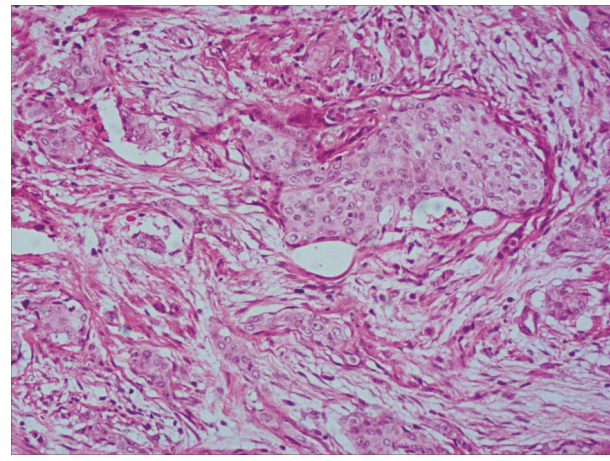

(c)

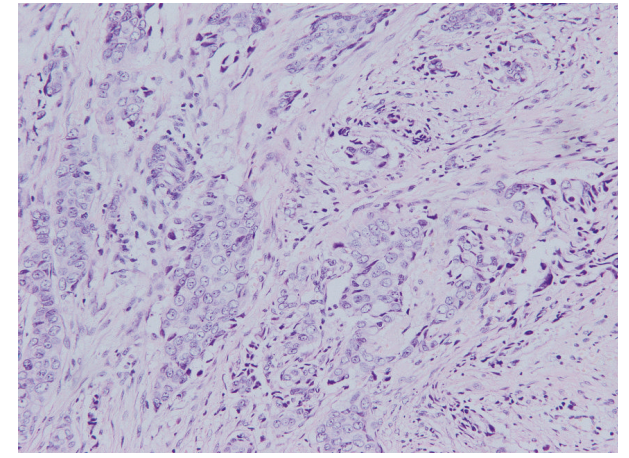

(b)

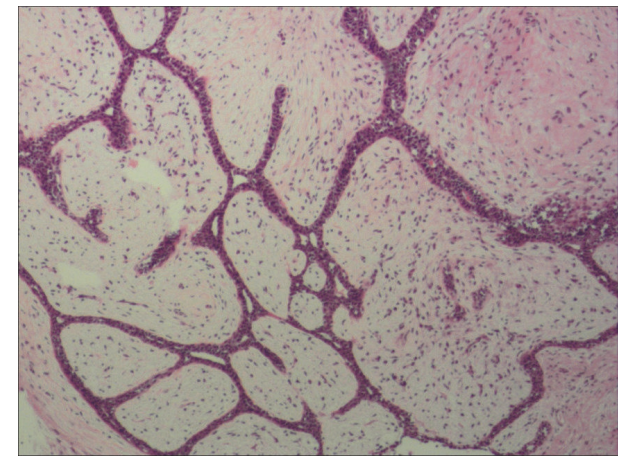

(d)

FIGURE 1: Histopathological diagnosis (H\&E staining) of samples from the 3 groups. (a) Breast cancer bone metastasis (200x). (b) Primary lesion of breast cancer with bone metastasis (200x), showing nonspecific invasive ductal carcinoma, WHO grade III. (c) Primary lesion of breast cancer without bone metastasis (200x), showing nonspecific invasive ductal carcinoma, WHO grade III. (d) Breast fibroadenoma $(200 x)$.

blocked with the 5\% normal goat serum for 1 hour at room temperature, the slides were incubated with the following primary antibodies at $4^{\circ} \mathrm{C}$ overnight: anti-TGF- $\alpha$ polyclonal antibody (ImmunoWay, Plano, TX, 1:50), antiCD68 antibody (Abcam, Cambridge, UK; 1:200), anti-IL6 antibody (ImmunoWay, Plano, TX, 1:50), anti-VEGF antibody (ImmunoWay, Plano, TX, 1:100), anti-FGF10 antibody (ABclonal, Boston, USA, 1:100), anti-FGF17 antibody (ImmunoWay, Plano, TX, $1: 100$ ), and anti-TGF- $\beta 1$ antibody (ABclonal, Boston, USA, 1:100). Following incubation with biotinylated secondary antibodies, the streptavidin-biotin complex/horseradish peroxidase was applied. Finally, the immunoreaction signal was developed with DAB staining, and the slides were counterstained in hematoxylin.

The stained tissue sections were reviewed under a light microscope (Nikon ECLIPSE Ni-U, Tokyo, Japan). The numbers of TGF- $\alpha$ and CD68 positive cells were counted in 5 random fields $(400 x)$ of paraneoplastic tissue of each tissue section, respectively.

2.3. Cell Culture. Human bone marrow-derived MSCs (hBMSCs) were purchased from Guangzhou Jenniobio Biotechnology Co., Ltd (Guangzhou, China), and cultured in DMEM medium (GIBCO, Gaithersburg, MD) supplemented with $10 \%$ fetal bovine serum (HyClone, Logan, UT) and $1 \%$ penicillin/streptomycin (Invitrogen, Waltham, MA) in a
$5 \% \mathrm{CO}_{2}$ humidified atmosphere at $37^{\circ} \mathrm{C}$. TGF- $\alpha$ exposure experiments were performed using hBMSCs between passages three and seven. Human BMSCs were treated for 8 or 24 hours with recombinant human TGF- $\alpha$ (Sino Biological, Beijing, China) at a concentration of 0 (medium control), 10, or $20 \mathrm{ng} / \mathrm{ml}$.

2.4. Quantitative PCR ( $q$-PCR). Total RNA was extracted from untreated or TGF $\alpha$-treated hBMSCs using Trizol reagent (Invitrogen), and cDNA was synthesized using an access RT system (Promega, Madison, WI). Real-time PCR was performed using ABI7500 Real-Time PCR System and SYBR Premix ExTaq II kit (TaKaRa Biotechnology Co., Ltd, Dalian, China). The primer sequences were selected as follows: IL-6, forward primer CATCCTCGACGGCATCTCAG and reverse primer ACCAGGCAAGTCTCCTCATTG; VEGF, forward primer CATCACCATGCAGATTATGCG$G$ and reverse primer GAGGCTCCAGGGCATTAGAC; fibroblast growth factor (FGF) 10, forward primer CCGTACAGCATCCTGGAGATAAC and reverse primer CCTCCCATTATGCTGCCAGTT; FGF 17, forward primer CCCAACCTCACTCTGTGCTTAC and reverse primer TGTAGAGTTGGTACTCGCGG; TGF- $\beta 1$, forward primer CGACTCGCCAGAGTGGTTAT and reverse primer GGTAGTGAACCCTGCGTTGAT. The PCR condition was $95^{\circ} \mathrm{C}$ for $30 \mathrm{~s}$, followed by 40 cycles of amplification $\left(95^{\circ} \mathrm{C}\right.$ for $5 \mathrm{~s}, 60^{\circ} \mathrm{C}$ 


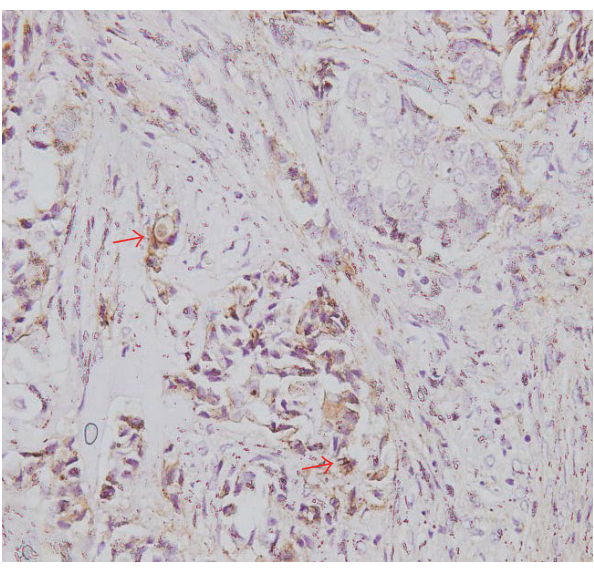

(a)

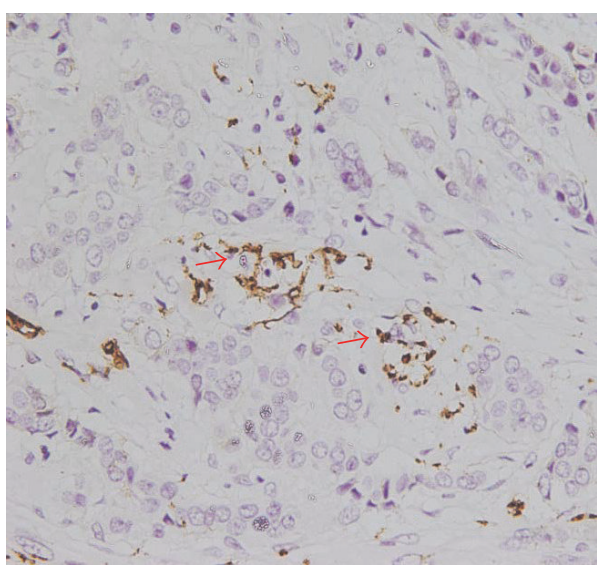

(c)

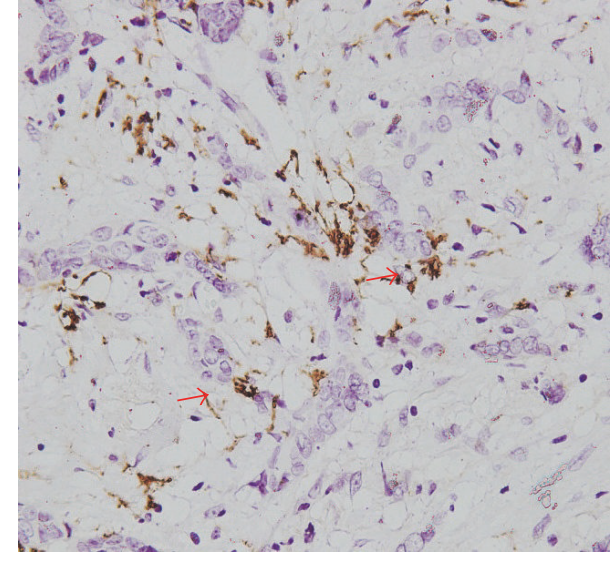

(b)

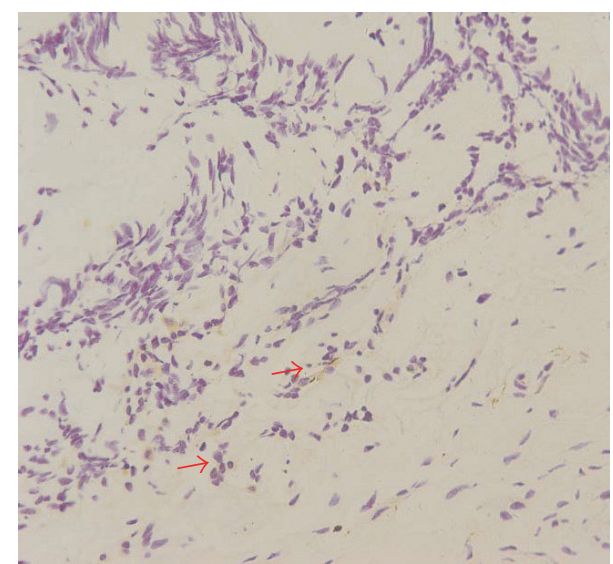

(d)

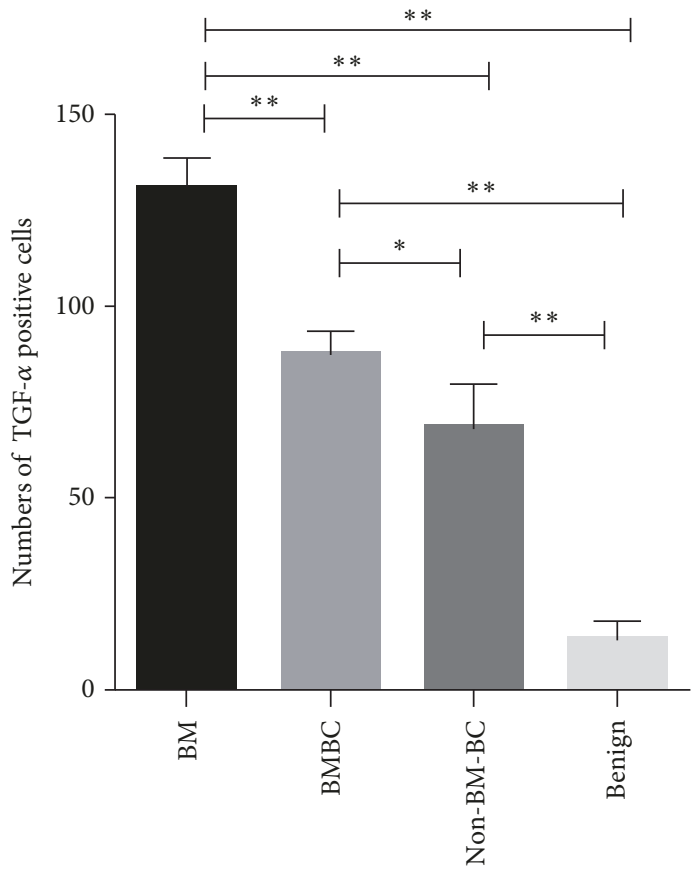

(e)

Figure 2: TGF- $\alpha$ in different tumor tissue. (a) TGF- $\alpha$ in bone metastasis (BM) lesion of breast cancer (400x), with arrows pointing to TGF- $\alpha$ positive cells; (b) TGF- $\alpha$ in primary lesion of breast cancer with bone metastasis (BMBC) (400x); (c) TGF- $\alpha$ in primary lesion of breast cancer without bone metastasis (Non-BM-BC) (400x); (d) TGF- $\alpha$ in benign breast fibroadenoma (400x); (e) comparison of TGF- $\alpha$ positive cells in tumor lesion of different groups (total positive cells in 5 random fields). ${ }^{*} P<0.05$; ${ }^{* *} P<0.01$. 


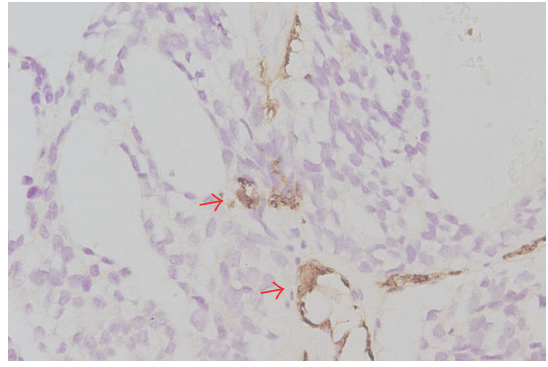

(a)

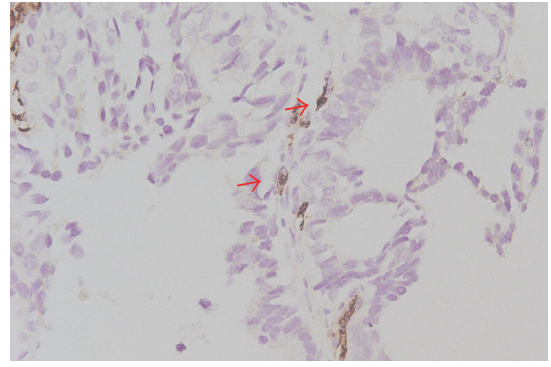

(b)

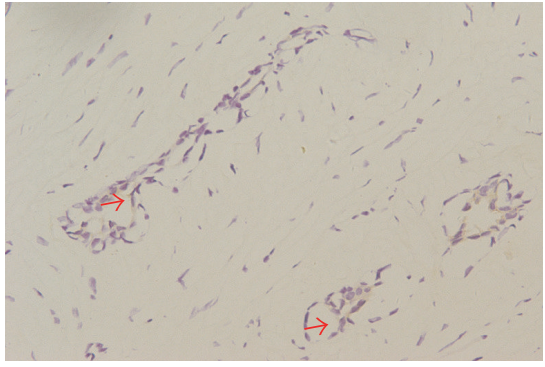

(c)

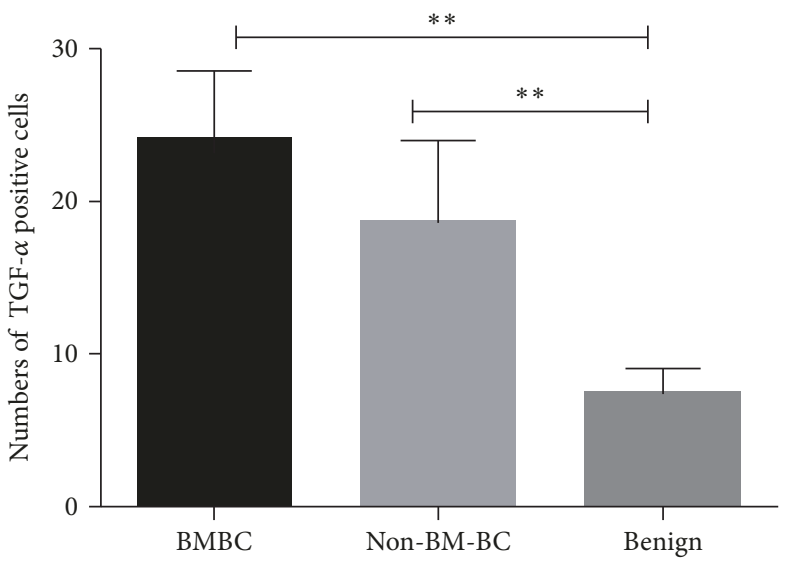

(d)

FiguRE 3: TGF- $\alpha$ expression in different nontumor tissues around tumor. (a) TGF- $\alpha$ in nontumor tissues around primary lesion of breast cancer with bone metastasis (BMBC) (400x, arrows pointing to TGF- $\alpha$ positive cells); (b) TGF- $\alpha$ in nontumor tissues around primary lesion of breast cancer without bone metastasis (Non-BM-BC) (400x); (c) TGF- $\alpha$ in nontumor tissues around benign breast fibroadenoma (400x); (d) comparison of TGF- $\alpha$ positive cells in nontumor tissues of different groups (total positive cells within 5 random fields). ${ }^{* *} P<0.01$.

for $34 \mathrm{~s}$, and $72^{\circ} \mathrm{C}$ for $34 \mathrm{~s}$ ). The relative quantification was determined using the $\Delta \Delta^{-\mathrm{Ct}}$ method and mRNA expression levels were normalized to internal control gene GAPDH. Each sample was tested three times.

2.5. Statistics. SPSS 19.0 (IBM SPSS Inc., Chicago, IL) was used to evaluate data. One-way analysis of variance followed by LSD $t$-test was used to analyze differences between groups, and 2-tailed significance was determined. Results are presented as the mean \pm standard deviation (SD) for all parameters measured. $P<0.05$ was considered statistically significant.

\section{Results}

3.1. TGF- $\alpha$ in Bone Metastasis and Primary Lesion of Breast Cancer and Benign Control. As analyzed by immunohistochemistry, bone metastases had a higher level TGF- $\alpha$ (more positive cells) than their own primary lesion $(P<0.01)$. TGF$\alpha$ level in primary lesion of BMBC group was higher than that in primary lesion of $\mathrm{BC}$ without bone metastasis $(P<0.05)$ (Figure 2). Benign control had a much lower level of TGF- $\alpha$ than the bone metastasis $(P<0.01)$ and all primary lesions $(P<0.01)$ (Figure 2).

Interestingly, TGF- $\alpha$ is expressed at higher levels in the nontumor tissues of both BMBC and Non-BM-BC while it was seldom found in nontumor tissues of the benign control $(P<0.01)$ (Figure 3). No significant differences were found in TGF- $\alpha$ levels in the nontumor tissues between BMBC and Non-BM-BC groups $(P>0.05)$ (Figure 3$)$.

\subsection{Macrophages (CD68+Cells) in BC Lesion and Nontumor} Tissues. Since macrophages are a known source of TGF$\beta 1$ in breast cancer, macrophages were detected by CD68 immunohistochemistry and positive cells were counted in $\mathrm{BC}$ lesion and nontumor tissues. Macrophages in BC lesion were found to be fewer than the nontumor tissue $(P<$ 0.01 ) (Figure 4). Numbers of macrophages in BC lesion were not different from those in benign lesion. Numbers of macrophages in nontumor tissue of BMBC and Non-BM-BC were higher than those in nontumor tissue of benign control $(P<0.01)$.

\subsection{Induction of Cytokines and Growth Factors from Human} BMSCs under TGF- $\alpha$ Stimulation and Their Increased Expression Levels in Different Nontumor Tissues around Tumor. Effects of TGF- $\alpha$ stimulation on expression of known genes involved in breast cancer metastasis were examined in human BMSCs. As analyzed by q-PCR, within 8 hours of TGF- $\alpha$ stimulation, expression of these procancer cytokines/growth factors was found to increase in a dose-dependent manner (Figure 5(a)). Within 24 hours, BMSCs with 20 ng/ml TGF- $\alpha$ 

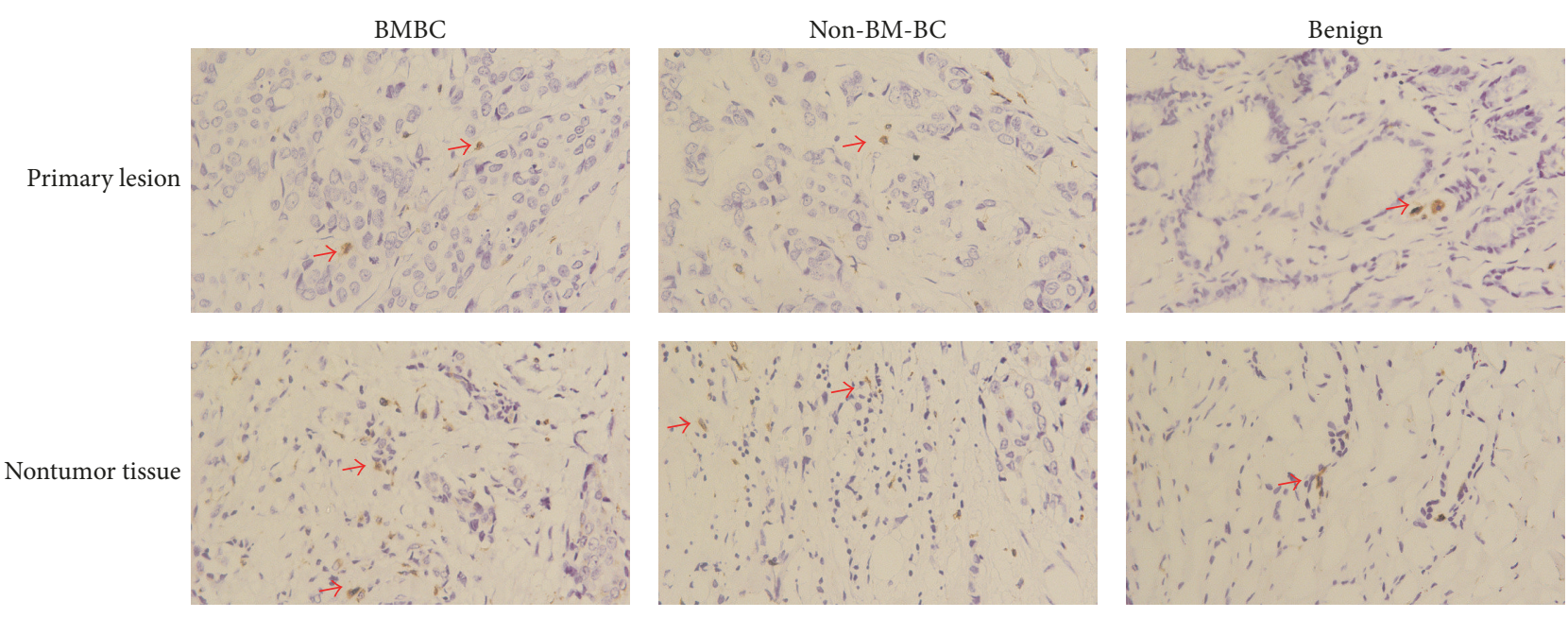

(a)

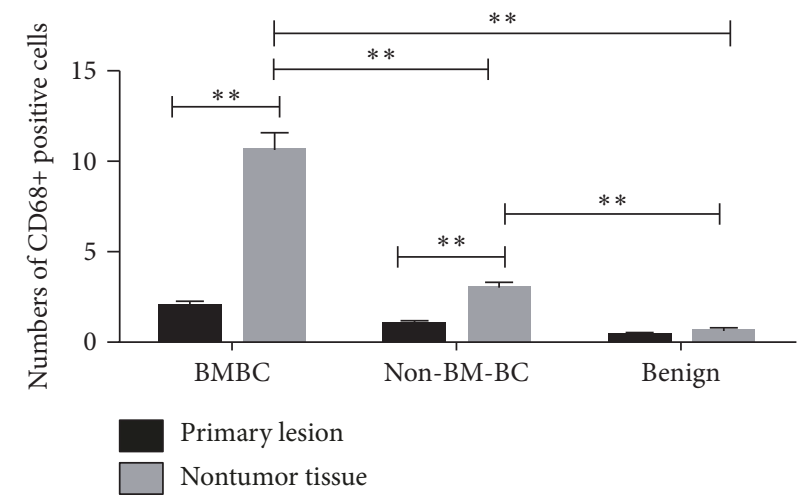

(b)

FIGURE 4: Numbers of macrophages (CD68+ cells) in different tissues. (a) Macrophages in primary lesion and nontumor tissues (400x, arrows indicating CD68+ positive cells); (b) comparison of macrophage numbers in different tissues. ${ }^{* *} P<0.01$.

stimulation produced significantly higher levels of IL-6 $(P<$ $0.05)$, VEGF $(P<0.05)$, FGF10 $(P<0.05)$, FGF17 $(P<0.05)$, and TGF- $\beta 1(P<0.05)$, compared with BMSCs with 0 or $10 \mathrm{ng} / \mathrm{ml}$ TGF- $\alpha$ treatment (Figure 5(b)). These cytokines are expressed at higher levels in the nontumor tissues of both $\mathrm{BMBC}$ and Non-BM-BC while they were seldom found in the benign control $(P<0.01)$ (Figures 5(c) and 5(d)). The level in the nontumor tissues of $\mathrm{BMBC}$ is higher than that of the Non-BM-BC group $(P<0.05)$ (Figures $5(\mathrm{c})$ and $5(\mathrm{~d}))$.

\section{Discussions}

Breast cancer bone metastasis in the early stage of primary lesion is threatening the patients at the rate of $20 \%$. The BMBC patients with removable bone metastasis like the 4 cases in the study were rare. Most of them developed unresectable multiple bone metastases [21-23]. While the interaction between BC cells and BMSCs has been reported to contribute to tumor angiogenesis, invasion, and thus metastasis [24, 25]; the signals (such as cytokines/growth factors) responsible for or underlying this interaction remain largely unclear. In the current study, our results showed that, in breast cancer bone metastasis, TGF- $\alpha$ might play a signaling role bridging $\mathrm{BC}$ cells and BMSCs and promote the bone metastasis.

In the human BMBC, TGF- $\alpha$ was found to be expressed in the bone metastasis lesion, at a higher level than that in primary lesion of BMBC and Non-BM-BC (Figure 2), and TGF- $\alpha$ level in primary lesion of BMBC and Non-BM-BC was found to be higher than that in Benign control. These findings suggest that TGF- $\alpha$ might be a special signal for breast cancer and its bone metastasis. In the malignant lesion, cancer cells could be the source of TGF- $\alpha$. In this study, TGF$\alpha$ was found to promote BMSCs to increase their transcription of IL-6, VEGF, FGF10, FGF 17 , and TGF- $\beta 1$ that could promote BC metastasis obviously (Figure 5). IL-6, VEGF, and TGF- $\beta 1$ have been proved to be commonly overexpressed in human breast cancer cases [26-28], FGF10 played the important role in type III epithelial-mesenchymal transition (EMT) on breast cancer cells and initiation of metastasis [29], and high expression of FGF17 causes tamoxifen resistance [30]. It could be inferred that overexpressed TGF- $\alpha$ in BC is an important signal that may bridge bone metastasis, $\mathrm{BC}$, and BMSCs. 


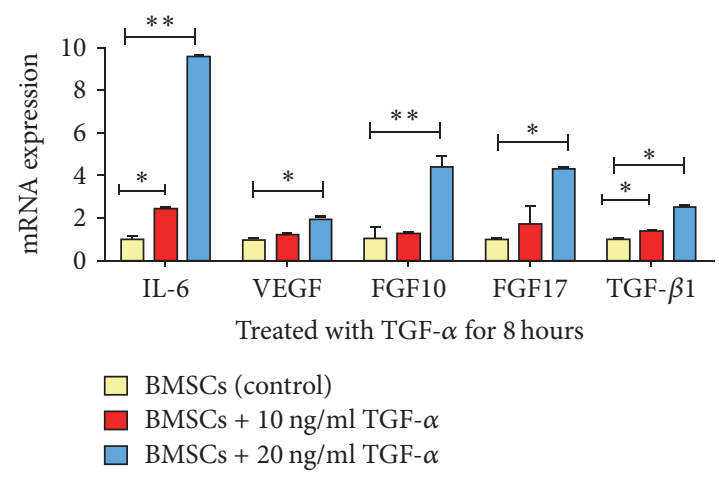

(a)
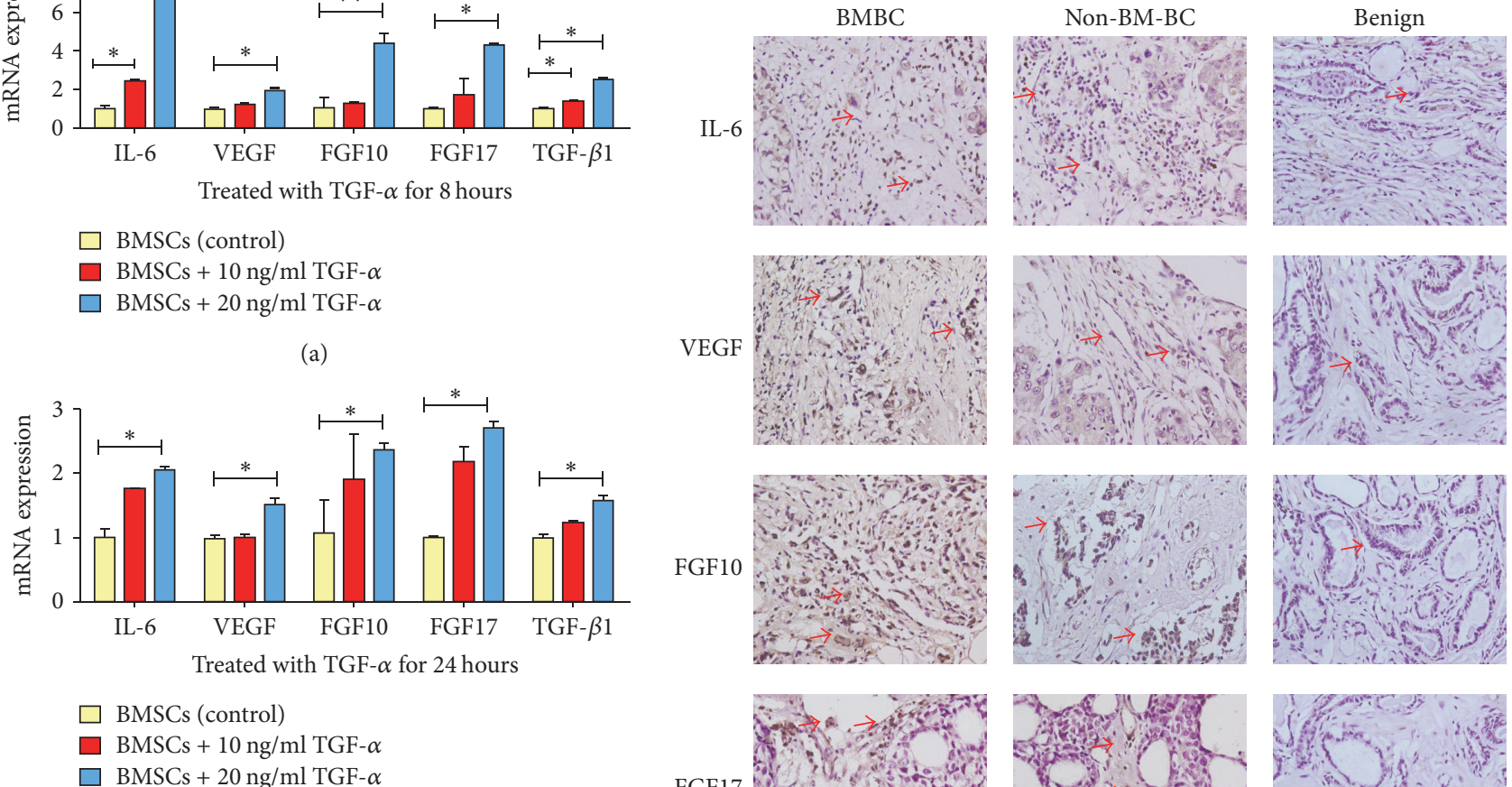

(b)
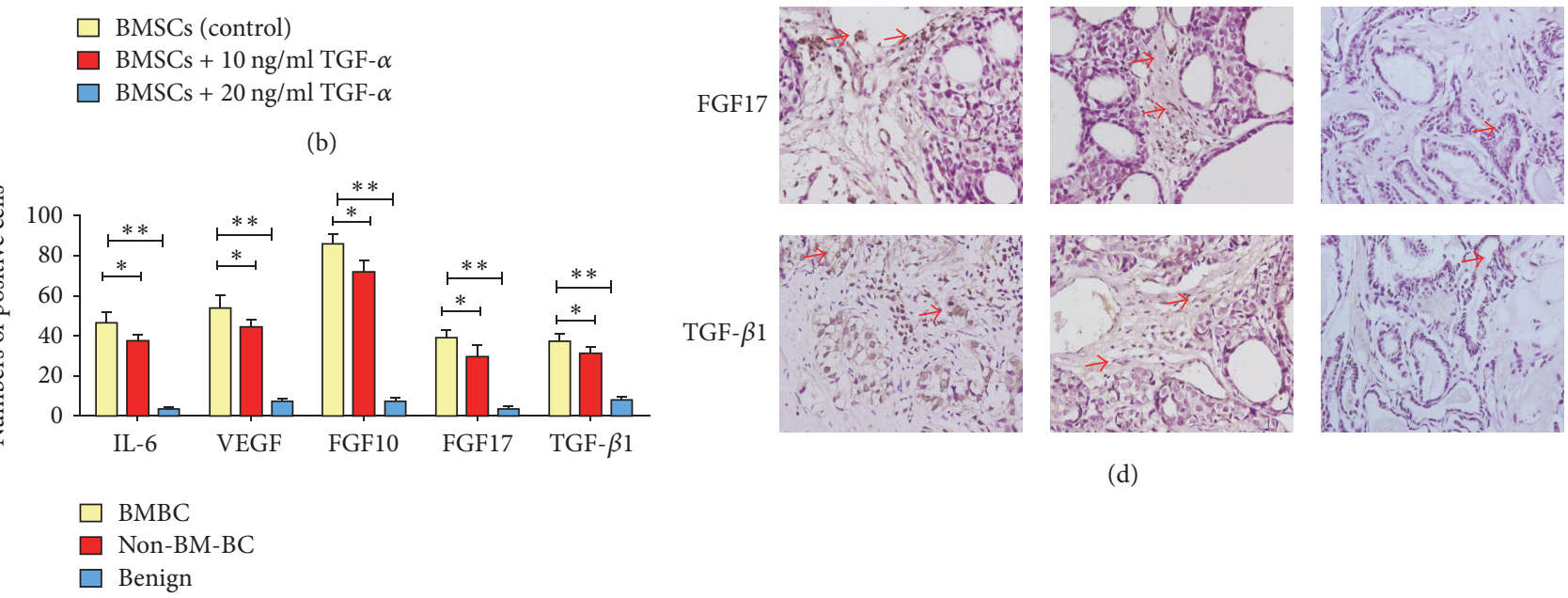

(d)

(c)

FIGURE 5: Changes in mRNA expression of cytokines and growth factors in BMSCs treated with different concentrations of TGF- $\alpha$. (a) 8 hours after treatment; (b) 24 hours after treatment; (c) comparison of cytokines and growth factors expression in different nontumor tissues around tumor. ${ }^{*} P<0.05 ;{ }^{* *} P<0.01$ compared with no treatment control; (d) cytokines and growth factors expression in different nontumor tissues around tumor (400x, arrows indicating positive cells).

Interestingly, in the human BMBC samples, TGF- $\alpha$ was also overexpressed in the nontumor tissue around the primary lesion when compared to those in benign control, but it was not expressed at a higher level than that of the nontumor tissue of Non-BM-BC (Figure 3). Our analyses on TGF- $\alpha$ expression in the "soil" of BC suggest that TGF$\alpha$ in the microenvironment might not be single signal in breast cancer bone metastasis. However, TGF- $\alpha$ in the microenvironment might play important roles in primary breast cancer development, and thus TGF- $\alpha$ in normal breast tissue might be a prognostic marker for predicting the risk of BC.
In the breast microenvironment, macrophages are an important component and known as a major cellular source of TGF- $\alpha[31,32]$. In the current study, macrophages were found at higher numbers in the nontumor tissue of $\mathrm{BC}$ than benign control (Figure 4). This could potentially explain the higher level of TGF- $\alpha$ in the nontumor tissue of BC. In the physiological situation, the interaction of macrophages and BMSCs has been recognized to be important in tissue regeneration during tissue repair, and TGF- $\alpha$ is the signal for interaction between macrophages and BMSCs in wound healing [33-36]. Bone metastasis frequently happens to the human loaded bones like the cases presented in the study, and 


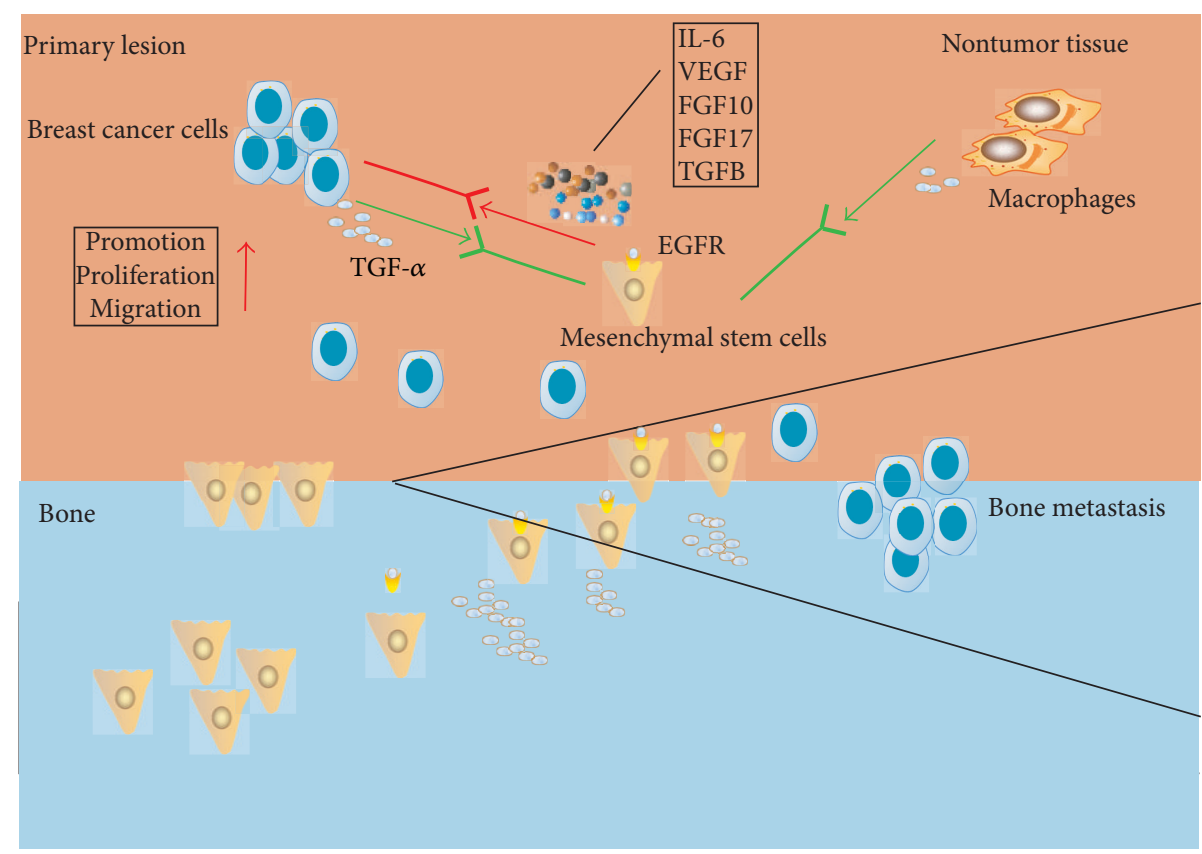

FIGURE 6: The hypothesis that TGF- $\alpha$ stimulates bone marrow-derived mesenchymal stem cells (BMSCs) in breast cancer bone metastasis. Breast cancer influences BMSCs to promote BC metastasis to bone. In the process, breast cancer cells and nontumor tissue macrophages produce TGF- $\alpha$ to stimulate BMSCs that express the receptor EGFR, and activated BMSCs produce procancer cytokines/growth factors to promote BC.

many risk factors could cause damage and subsequent repair in the human loaded bones [37, 38]. With the above known roles of TGF- $\alpha$ and our finding of overexpressed TGF- $\alpha$ being found mostly in the bone metastasis lesion in the study (Figure 2), it is possible that $\mathrm{BC}$ with elevated expression of TGF- $\alpha$ might mimic the macrophages in tissue regeneration to influence BMSCs to promote BC to metastasis (Figure 6). On the other hand, as the source of TGF- $\alpha$, macrophages in the nontumor tissue of $\mathrm{BC}$ may play a more procancer role than an anticancer role.

In summary, TGF- $\alpha$ in BMBC lesion was found to be overexpressed and could be an important signal. TGF- $\alpha$ in $\mathrm{BC}$ lesion and from nontumor tissue could stimulate BMSCs to promote cancer metastasis. In the further study, more operable BMBC cases would be enrolled. Protein analyses would be performed to define the precise role of TGF- $\alpha$ in breast cancer secretion. BMSCs transfected by no-load virus red fluorescent protein would be used in in vivo models to evaluate the action mechanism of TGF- $\alpha$ locally expressed in $\mathrm{BC}$ lesion in influencing the BMSCs and in promoting bone metastasis, information from which could lead to therapeutic targets by controlling TGF- $\alpha$ signaling in both primary lesion and the surrounding microenvironment.

\section{Disclosure}

The first corresponding author is Xiaolong Liu and cocorresponding author is Lixin Liu.

\section{Conflicts of Interest}

All authors declare that they have no conflicts of interest.

\section{Authors' Contributions}

Jingbo Sun and Haiyan Cui contributed equally to the work. Xiaolong Liu, Lixin Liu, Jingbo Sun, and Haiyan Cui were responsible for study design. Jingbo Sun and Yanxin Gao conducted the study. Jingbo Sun, Xiaolong Liu, Yanxin Gao, and Haiyan Cui collected the data. Xiaolong Liu, Jingbo Sun, Yanxin Gao, and Haiyan Cui analyzed the data. Cory J. Xian, Xiaolong Liu, Jingbo Sun, Yanxin Gao, Yangjian Pan, Kun Zhou, Jingzhan Huang, Jin Lan, and Haiyan Cui were responsible for data interpretation. Xiaolong Liu, Jingbo Sun, Haiyan Cui, Yanxin Gao, Yangjian Pan, Kun Zhou, Jingzhan Huang, Jin Lan, and Lixin Liu drafted the manuscript. Cory J. Xian, Xiaolong Liu, and Lixin Liu revised the manuscript. All authors have read and approved the final submitted manuscript.

\section{Acknowledgments}

The authors would like to thank Professor Li Liang of the Key Laboratory of Molecular Tumor Pathology in Guangdong Province for her instructions on pathological analyses. This work was supported by the Science and Technology Plan Project of Tianhe District (201604KW011). Cory J. Xian is funded by NHMRC Senior Research Fellowship (1042105). 


\section{References}

[1] K. Manders, L. V. van de Poll-Franse, G.-J. Creemers et al., "Clinical management of women with metastatic breast cancer: A descriptive study according to age group," BMC Cancer, vol. 6, 179 pages, 2006.

[2] L. A. Torre, F. Bray, R. L. Siegel, J. Ferlay, and J. LortetTieulent, "Global cancer statistics, 2012," CA: A Cancer Journal for Clinicians, vol. 65, no. 2, pp. 87-108, 2015.

[3] S. Madar, I. Goldstein, and V. Rotter, "Cancer associated fibroblasts'-more than meets the eye," Trends in Molecular Medicine, vol. 19, no. 8, pp. 447-453, 2013.

[4] K. velaei, N. Samadi, B. Barazvan, and J. Soleimani Rad, “Tumor microenvironment-mediated chemoresistance in breast cancer," The Breast, vol. 30, pp. 92-100, 2016.

[5] D. L. Alge, D. Zhou, L. L. Adams et al., "Donor-matched comparison of dental pulp stem cells and bone marrow-derived mesenchymal stem cells in a rat model," Journal of Tissue Engineering \& Regenerative Medicine, vol. 4, no. 1, pp. 73-81, 2010.

[6] A. Erices, P. Conget, and J. J. Minguell, "Mesenchymal progenitor cells in human umbilical cord blood," British Journal of Haematology, vol. 109, no. 1, pp. 235-242, 2000.

[7] P. A. Zuk, M. Zhu, P. Ashjian et al., "Human adipose tissue is a source of multipotent stem cells," Molecular Biology of the Cell (MBoC), vol. 13, no. 12, pp. 4279-4295, 2002.

[8] Y. Zheng, W. Cai, S. Zhou, L. Xu, and C. Jiang, "Protective effect of bone marrow derived mesenchymal stem cells in lipopolysaccharide-induced acute lung injury mediated by claudin-4 in a rat model," American Journal of Translational Research, vol. 8, no. 9, pp. 3769-3779, 2016.

[9] F. Gao, S. M. Chiu, D. A. Motan et al., "Mesenchymal stem cells and immunomodulation: current status and future prospects," Cell Death \& Disease, vol. 7, no. 1, article e2062, 2016.

[10] G. O’Malley, M. Heijltjes, A. M. Houston et al., "Mesenchymal stromal cells (MSCs) and colorectal cancer - A troublesome twosome for the anti-tumour immune response?" Oncotarget , vol. 7, no. 37, pp. 60752-60774, 2016.

[11] A. De Luca, L. Lamura, M. Gallo, V. Maffia, and N. Normanno, "Mesenchymal stem cell-derived interleukin-6 and vascular endothelial growth factor promote breast cancer cell migration," Journal of Cellular Biochemistry, vol. 113, no. 11, pp. 3363-3370, 2012.

[12] K. M. McAndrews, D. J. McGrail, N. Ravikumar, and M. R. Dawson, "Mesenchymal Stem Cells Induce Directional Migration of Invasive Breast Cancer Cells through TGF- $\beta$," Scientific Reports, vol. 5, Article ID 16941, 2015.

[13] T. J. Bartosh, M. Ullah, S. Zeitouni, J. Beaver, and D. J. Prockop, "Cancer cells enter dormancy after cannibalizing mesenchymal stem/stromal cells (MSCs)," Proceedings of the National Acadamy of Sciences of the United States of America, vol. 113, no. 42, pp. E6447-E6456, 2016.

[14] A. De Luca, C. Roma, M. Gallo et al., "RNA-seq analysis reveals significant effects of EGFR signalling on the secretome of mesenchymal stem cells," Oncotarget , vol. 5, no. 21, pp. 1051810528, 2014.

[15] I. Ferrer, S. Alcántara, J. Ballabriga et al., "Transforming growth factor- $\alpha$ (TGF- $\alpha$ ) and epidermal growth factor-receptor (EGF$\mathrm{R}$ ) immunoreactivity in normal and pathologic brain," Progress in Neurobiology, vol. 49, no. 2, pp. 99-123, 1996.
[16] S. Raja, G. Byakod, and P. Pudakalkatti, "Growth factors in periodontal regeneration," International Journal of Dental Hygiene, vol. 7, no. 2, pp. 82-89, 2009.

[17] C. J. Xian, "Roles of epidermal growth factor family in the regulation of postnatal somatic growth," Endocrine Reviews, vol. 28 , no. 3, pp. 284-296, 2007.

[18] R. J. Coffey, M. K. Washington, C. L. Corless, and M. C. Heinrich, "Ménétrier disease and gastrointestinal stromal tumors: Hyperproliferative disorders of the stomach," The Journal of Clinical Investigation, vol. 117, no. 1, pp. 70-80, 2007.

[19] J. A. Schroeder, K. L. Troyer, and D. C. Lee, "Cooperative induction of mammary tumorigenesis by TGF $\alpha$ and Wnts," Oncogene, vol. 19, no. 28, pp. 3193-3199, 2000.

[20] A. Orucevic, J. Chen, J. M. McLoughlin, R. E. Heidel, T. Panella, and J. Bell, "Is the TNM staging system for breast cancer still relevant in the era of biomarkers and emerging personalized medicine for breast cancer - An institution's 10-year experience," The Breast Journal, vol. 21, no. 2, pp. 147-154, 2015.

[21] X. Lu and Y. Kang, "Organotropism of breast cancer metastasis," Journal of Mammary Gland Biology and Neoplasia, vol. 12, no. 23, pp. 153-162, 2007.

[22] K. R. Hess, G. R. Varadhachary, S. H. Taylor et al., "Metastatic patterns in adenocarcinoma," Cancer, vol. 106, no. 7, pp. 16241633, 2006.

[23] T. A. Guise, W. M. Kozlow, A. Heras-Herzig, S. S. Padalecki, J. J. Yin, and J. M. Chirgwin, "Molecular mechanisms of breast cancer metastases to bone," Clinical Breast Cancer, vol. 5, Supplement 2, pp. S46-S53, 2005.

[24] A. E. Karnoub, A. B. Dash, A. P. Vo et al., "Mesenchymal stem cells within tumour stroma promote breast cancer metastasis," Nature, vol. 449, no. 7162, pp. 557-563, 2007.

[25] F. T. Martin, R. M. Dwyer, J. Kelly et al., "Potential role of mesenchymal stem cells (MSCs) in the breast tumour microenvironment: Stimulation of epithelial to mesenchymal transition (EMT)," Breast Cancer Research and Treatment, vol. 124, no. 2, pp. 317-326, 2010.

[26] A. S. Noman, M. Uddin, A. A. Chowdhury et al., "Serum sonic hedgehog (SHH) and interleukin-(IL-6) as dual prognostic biomarkers in progressive metastatic breast cancer," Scientific Reports, vol. 7, no. 1, 1796 pages, 2017.

[27] H. Linardou, K. T. Kalogeras, R. Kronenwett et al., "The prognostic and predictive value of mRNA expression of vascular endothelial growth factor family members in breast cancer: A study in primary tumors of high-risk early breast cancer patients participating in a randomized Hellenic Cooperative Oncology Group trial," Breast Cancer Research, vol. 14, no. 6, article no. R145, 2012.

[28] E. M. de Kruijf et al., "The prognostic role of TGF-beta signaling pathway in breast cancer patients," Ann Oncol, vol. 24, no. 2, pp. 384-390, 2013.

[29] A. Abolhassani, G. H. Riazi, E. Azizi et al., "FGF10: Type III epithelial mesenchymal transition and invasion in breast cancer cell lines," Journal of Cancer, vol. 5, no. 7, pp. 537-547, 2014.

[30] D. Meijer, A. M. Sieuwerts, M. P. Look, T. Van Agthoven, J. A. Foekens, and L. C. J. Dorssers, "Fibroblast growth factor receptor 4 predicts failure on tamoxifen therapy in patients with recurrent breast cancer," Endocrine-Related Cancer, vol. 15, no. 1, pp. 101-111, 2008.

[31] S. Su, Q. Liu, J. Chen et al., "A positive feedback loop between mesenchymal-like cancer cells and macrophages is essential to breast cancer metastasis," Cancer Cell, vol. 25, no. 5, pp. 605620, 2014. 
[32] D. K. Madtes, E. W. Raines, K. S. Sakariassen et al., "Induction of transforming growth factor- $\alpha$ in activated human alveolar macrophages," Cell, vol. 53, no. 2, pp. 285-293, 1988.

[33] M. J. Lee, M. Y. Kim, S. C. Heo et al., "Macrophages regulate smooth muscle differentiation of mesenchymal stem cells via a prostaglandin $\mathrm{F}_{2 \alpha}$-mediated paracrine mechanism," Arteriosclerosis, Thrombosis, and Vascular Biology, vol. 32, no. 11, pp. 27332740, 2012.

[34] Y.-H. Kim and Y. Tabata, "Recruitment of mesenchymal stem cells and macrophages by dual release of stromal cell-derived factor-1 and a macrophage recruitment agent enhances wound closure," Journal of Biomedical Materials Research Part A, vol. 104, no. 4, pp. 942-956, 2016.

[35] G. Di, X. Du, X. Qi et al., "Mesenchymal stem cells promote diabetic corneal epithelial wound healing through TSG-6dependent stem cell activation and macrophage switch," Investigative Ophthalmology \& Visual Science, vol. 58, no. 10, pp. 4344-4354, 2017.

[36] S.-Y. Cho, E.-Y. Kwon, S.-M. Choi et al., "Immunomodulatory effect of mesenchymal stem cells on the immune response of macrophages stimulated by Aspergillus fumigatus conidia," Medical Mycology, vol. 54, no. 4, pp. 377-383, 2016.

[37] M. Hassanshahi, A. Hassanshahi, S. Khabbazi, Y.-W. Su, and C. J. Xian, "Bone marrow sinusoidal endothelium: damage and potential regeneration following cancer radiotherapy or chemotherapy," Angiogenesis, pp. 1-16, 2017.

[38] C. Fan, K. R. Georgiou, H. A. Morris et al., "Combination breast cancer chemotherapy with doxorubicin and cyclophosphamide damages bone and bone marrow in a female rat model," Breast Cancer Research and Treatment, vol. 165, no. 1, pp. 41-51, 2017. 


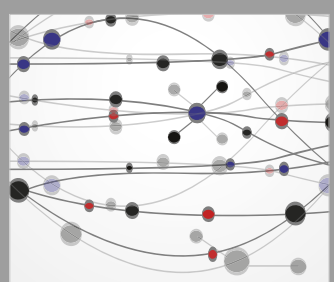

The Scientific World Journal
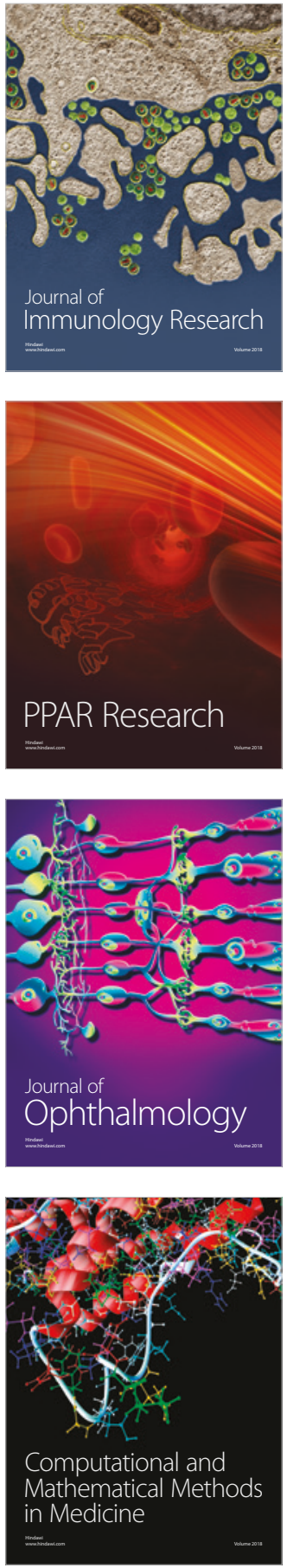

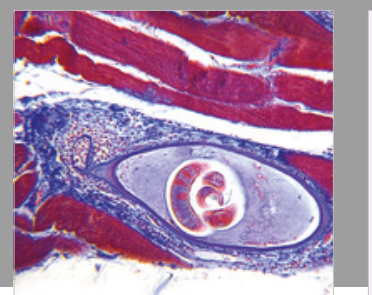

Gastroenterology Research and Practice

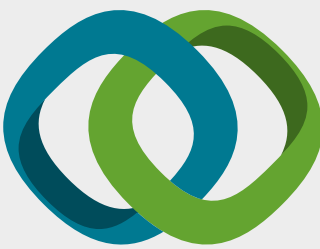

\section{Hindawi}

Submit your manuscripts at

www.hindawi.com
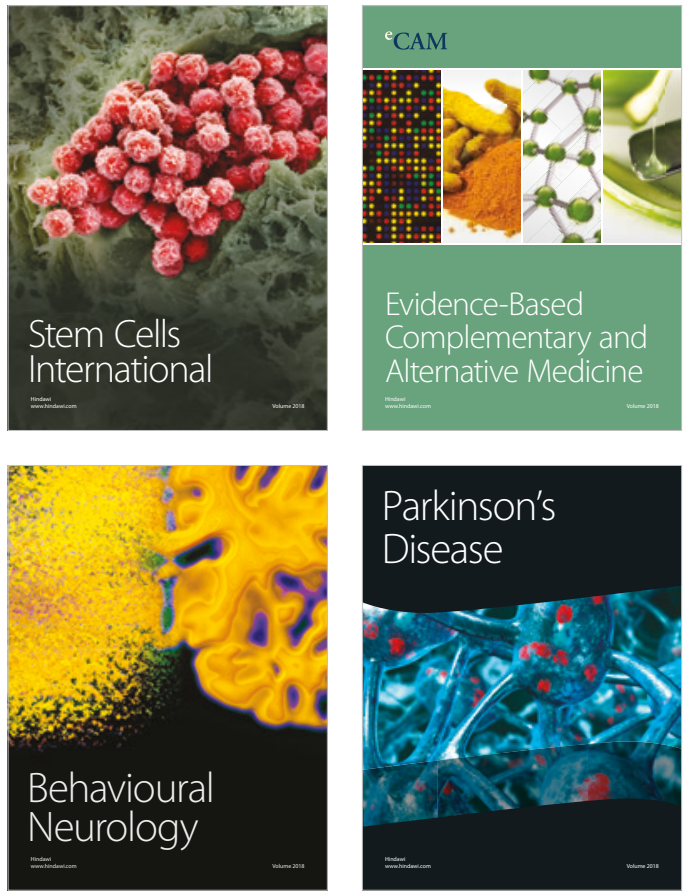

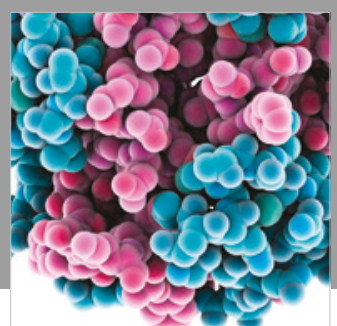

ournal of

Diabetes Research

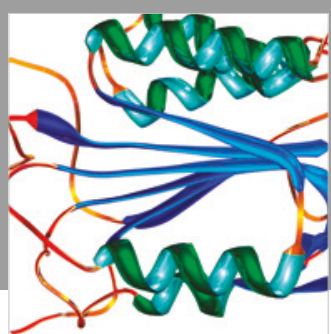

Disease Markers
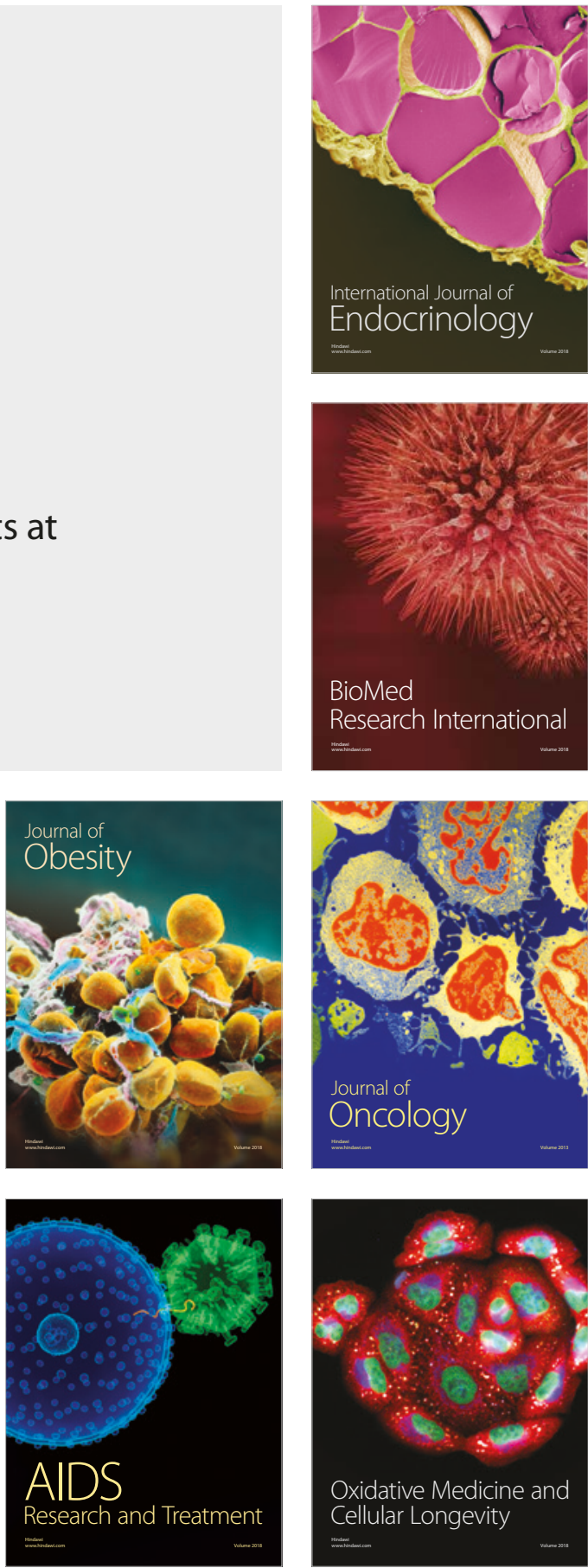